\title{
Erratum to: costs of major intracranial, gastrointestinal and other bleeding events in patients with atrial fibrillation - a nationwide cohort study
}

Marie Jakobsen ${ }^{1 *}$, Christophe Kolodziejczyk ${ }^{1}$, Eskild Klausen Fredslund ${ }^{1}$, Peter Bo Poulsen², Lars Dybro ${ }^{2}$ and Søren Paaske Johnsen ${ }^{3}$

\section{Erratum}

Following publication of the original article [1], the authors asked for the first formula to be changed from.

Atributable costs i $t=(C d t \backslash$ colorbox $[r g b] 1,1,0 \$-\$ C$ $d-1)-(C \operatorname{ct}-C c-1)$.

to

$$
\text { Atributable costs }{ }_{t}^{i}=\left(C_{t}^{d}-C_{-1}^{d}\right)-\left(C_{t}^{c}-C_{-1}^{c}\right),
$$

The original article has been corrected.

\section{Author details}

'KORA, Danish Institute for Local and Regional Government Research, Købmagergade 22, DK-1150 København K, Denmark. ${ }^{2}$ Pfizer Denmark, Lautrupvang 8, DK-2750 Ballerup, Denmark. ${ }^{3}$ Department of Clinical

Epidemiology, Aarhus University Hospital, Olofs Palmes Allé 43-45, DK-8200 Aarhus N, Denmark.

Received: 12 September 2017 Accepted: 12 September 2017

Published online: 09 October 2017

\section{Reference}

1. Jakobsen M, Kolodziejczyk C, Fredslund EK, Poulsen PB, Dybro L, Johnsen

SP. Costs of major intracranial, gastrointestinal and other bleeding events in patients with atrial fibrillation - a nationwide cohort study. BMC Health Serv Res. 2017;17:398. doi: 10.1186/s12913-017-2331-z.

\footnotetext{
* Correspondence: maja@kora.dk

'KORA, Danish Institute for Local and Regional Government Research,

Købmagergade 22, DK-1150 København K, Denmark
} 